\title{
Predicting the structure of soil communities from plant community taxonomy, phylogeny, and traits
}

\author{
Jonathan W. Leff ${ }^{1,2} \cdot$ Richard D. Bardgett $^{3} \cdot$ Anna Wilkinson $^{3} \cdot$ Benjamin G. Jackson $^{4} \cdot$ William J. Pritchard ${ }^{3}$. \\ Jonathan R. De Long $\mathbb{D}^{3} \cdot$ Simon Oakley ${ }^{5}$. Kelly E. Mason ${ }^{5} \cdot$ Nicholas J. Ostle $^{6} \cdot$ David Johnson $^{3}$. \\ Elizabeth M. Baggs ${ }^{7} \cdot$ Noah Fierer $^{1,2}$
}

Received: 17 September 2017 / Revised: 14 January 2018 / Accepted: 20 January 2018 / Published online: 9 March 2018

(c) International Society for Microbial Ecology 2018

\begin{abstract}
There are numerous ways in which plants can influence the composition of soil communities. However, it remains unclear whether information on plant community attributes, including taxonomic, phylogenetic, or trait-based composition, can be used to predict the structure of soil communities. We tested, in both monocultures and field-grown mixed temperate grassland communities, whether plant attributes predict soil communities including taxonomic groups from across the tree of life (fungi, bacteria, protists, and metazoa). The composition of all soil community groups was affected by plant species identity, both in monocultures and in mixed communities. Moreover, plant community composition predicted additional variation in soil community composition beyond what could be predicted from soil abiotic characteristics. In addition, analysis of the field aboveground plant community composition and the composition of plant roots suggests that plant community attributes are better predictors of soil communities than root distributions. However, neither plant phylogeny nor plant traits were strong predictors of soil communities in either experiment. Our results demonstrate that grassland plant species form specific associations with soil community members and that information on plant species distributions can improve predictions of soil community composition. These results indicate that specific associations between plant species and complex soil communities are key determinants of biodiversity patterns in grassland soils.
\end{abstract}

Electronic supplementary material The online version of this article (https://doi.org/10.1038/s41396-018-0089-x) contains supplementary material, which is available to authorized users.

Noah Fierer

Noah.Fierer@colorado.edu

1 Cooperative Institute for Research in Environmental Sciences, University of Colorado, Boulder, CO 80309, USA

2 Department of Ecology and Evolutionary Biology, University of Colorado, Boulder, CO 80309, USA

3 School of Earth and Environmental Sciences, Michael Smith Building, The University of Manchester, Oxford Road, Manchester M13 9PT, UK

4 School of Geosciences, Grant Institute, The King's Buildings, James Hutton Road, Edinburgh EH9 3FE, UK

5 Centre for Ecology \& Hydrology, Lancaster Environment Centre, Library Avenue, Bailrigg, Lancaster LA1 4AP, UK

6 Lancaster Environment Centre, Lancaster University, Lancaster LA1 4YQ, UK

7 The Royal (Dick) School of Veterinary Studies, University of Edinburgh, Easter Bush Campus Buildings, Midlothian EH25 9RG, UK

\section{Introduction}

The interactions between plants and soil organisms can have important ramifications for ecosystem functioning and plant community dynamics, but the extent to which these interactions influence the spatial distributions of soil communities remains poorly understood. Knowing how plants control the spatial variation in belowground communities is important for building a predictive understanding of the heterogeneity in soil communities and contributing to preexisting research that has identified how certain site and abiotic soil properties can influence the spatial variation in soil communities across large geographic scales [1-4]. Further, this information will aid our ability to probe the undescribed and likely diverse ways in which soil organisms interact with plants since comparatively few plant-microbe interactions are well understood [5].

Certain soil organisms are known to form close associations with particular plant species [6,7]. Mycorrhizal relationships, for instance, involve a direct exchange of nutrients between plants and symbiotic soil fungi, and these 
relationships can influence plant-soil diversity linkages [8, 9]. Indirect mechanisms, such as the release of root exudates and microbial attraction to those exudates, can also drive associations between specific microbes and plant species [10]. However, these described interactions are likely only a small fraction of the numerous interactions among plants and soil organisms in a given ecosystem. Thus, it is uncertain whether the composition of soil communities as a whole is associated with plant community attributes under field conditions.

It has long been known that individual plant species can exert a powerful influence on soil microbial communities [11-13], and there is evidence that divergence in soil bacterial and fungal communities is broadly linked to plant community composition at landscape [14, 15] and global scales [16]. Additionally, correlational analyses have revealed associations between individual plant species and soil fungal [17], bacterial [18], nematode [19], and arthropod [20] communities. However, it is unclear whether these relationships are driven by shared environmental preferences or by the direct effects of locally dominant plant species on soil communities. While plant invasions can elicit shifts in soil community structure [21, 22], the effects of plant species identity on the overall composition of belowground communities are often weak or difficult to quantify, with several studies having failed to identify strong links between changes in plant assemblages and corresponding changes in soil communities [17, 23-26]. As such, the existence of a general relationship between plants and soil communities remains uncertain and difficult to predict a priori.

There are multiple plant community attributes that could potentially be used to predict variation in soil communities. Plant species identity could be a strong predictor of variation in soil communities $[11,17,19]$, as could evolutionary history (i.e. the phylogeny) of plants, given the potential for more closely related plants to be associated with more similar belowground communities [27]. Such patterns could arise as a product of coevolution between plants and soil microbes or if phylogenetic relatedness corresponds to other plant attributes that affect soil organisms [28]. It has also been proposed that plant functional traits could be used to predict plant-microbe associations a priori given that plant species' distributions and community diversity are generally predictable based on their traits [29, 30], and soil communities can form associations with plants based on these traits [6]. Although previous studies have shown that plant traits can explain variation in soil microbial processes involved in $\mathrm{C}$ and $\mathrm{N}$ cycling [31-35], it remains unclear whether variation in soil community composition is directly caused by, or merely associated with, differences in plant traits. Further, past studies show that links between plant traits and the composition of soil communities are not always observed
[27] and when they have been found, they are often based on crude assessments of microbial community composition, such as the relative abundance of fungi and bacteria [15, 35]. Likewise, most previous work has focused on the relationships between soil biota and aboveground plant traits, despite increasing evidence that root traits are likely to play a more important role in structuring belowground communities [36-38].

Here we provide the first in-depth evaluation of the predictive power of plant community attributes, alongside abiotic factors, for explaining spatial (i.e. horizontal) variation in soil communities at the individual plant and community scale. While previous work has investigated effects of plant species and community attributes on soil communities, we are not aware of any previous study that has comprehensively assessed these effects across such a wide range of functionally important belowground taxonomic groups. Specifically, we address the overarching question: Can plant community attributes (i.e. taxonomic composition, phylogenetic composition, and plant functional traits) be used to predict spatial variability in soil community composition? To address this question, we sampled soils from both monocultures of 21 common temperate grassland plant species spanning eight families and a range of life history strategies, and we sampled an adjacent field experiment where grassland community composition had been manipulated through plant species additions to create a gradient of plant species and plant functional diversity. We used DNA sequencing-based approaches to target soil fungal, bacterial, protistan, and metazoan (faunal) communities. We first assessed whether the identity, phylogenetic history, and/or functional traits of individual plant species (both leaf and root traits) could be used to explain variation in soil communities. Next, we determined whether observations made at the individual plant scale correspond to similar trends in mixed plant communities in the field.

\section{Materials and methods}

\section{Mesocosm experiment}

To evaluate effects of individual plant species, their phylogeny, and their functional traits on soil communities, mesocosms containing plants grown in monoculture were established in a fenced enclosure at Colt Park within the Ingleborough National Nature Reserve in England (54 $\left.11^{\prime} 38.7^{\prime \prime} \mathrm{N} 2^{\circ} 20^{\prime} 54.4^{\prime \prime} \mathrm{W}\right)$. Mesocosms were constructed from polypropylene pots $(38 \times 38 \times 30 \mathrm{~cm})$ filled with 10 $\mathrm{cm}$ of rinsed gravel and $20 \mathrm{~cm}$ sieved and homogenized top soil ( $\mathrm{pH} \sim 5.8 ; 8.9 \mathrm{C} \%$; $0.92 \mathrm{~N} \%$ ). Top soil was a brown earth sourced from the adjacent grassland, a mesotrophic 
A

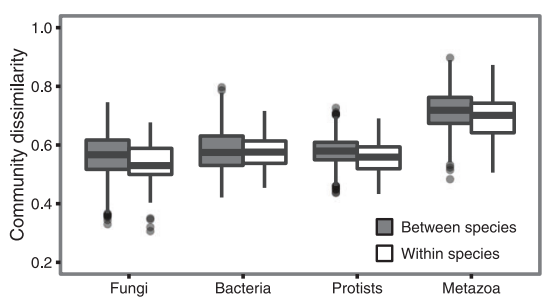

B
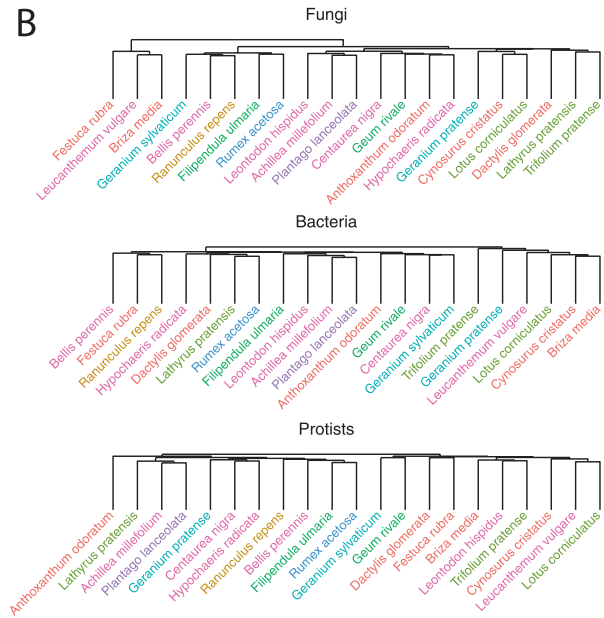

Metazoa

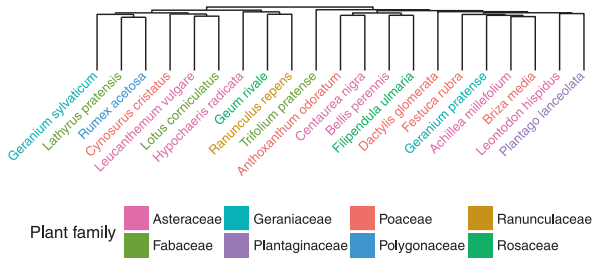

C

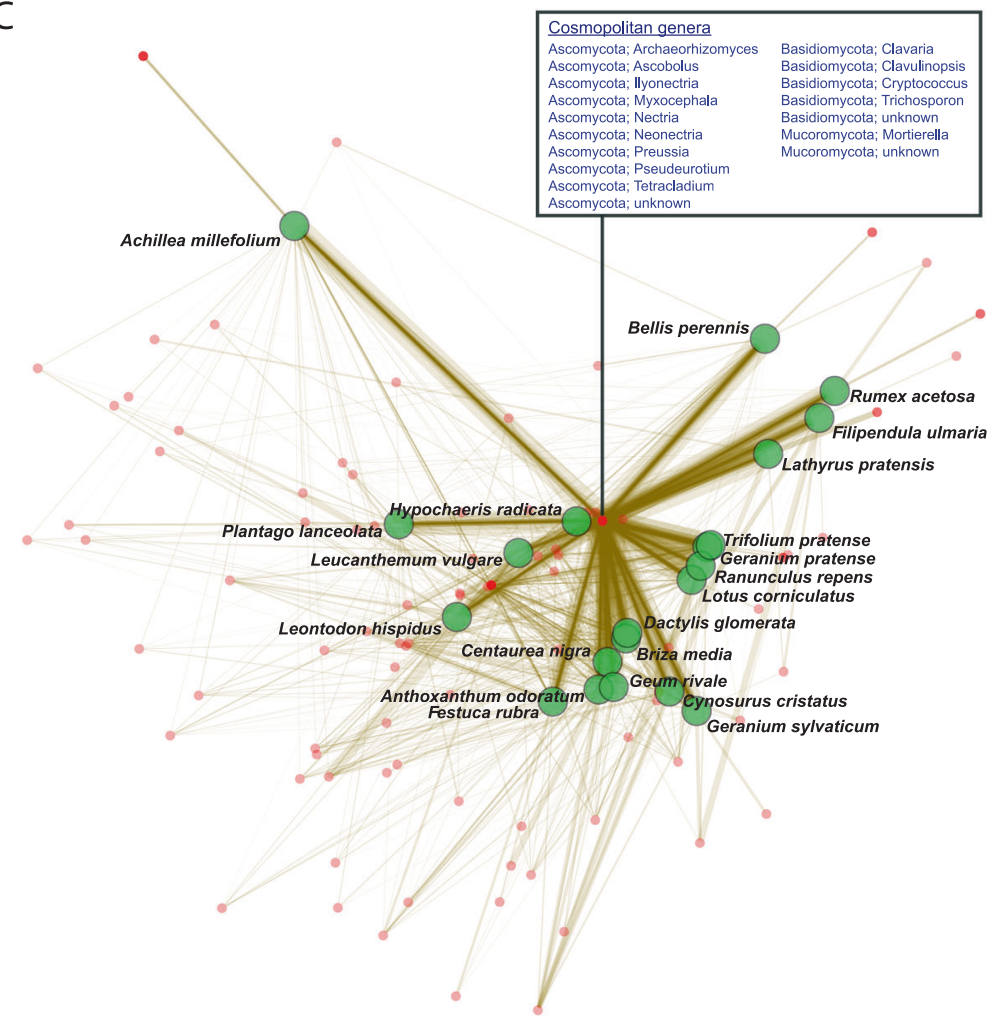

$\mathrm{D}$

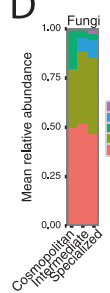

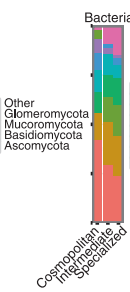

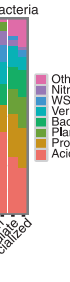

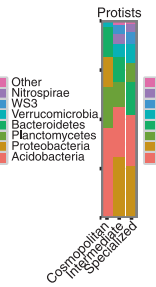
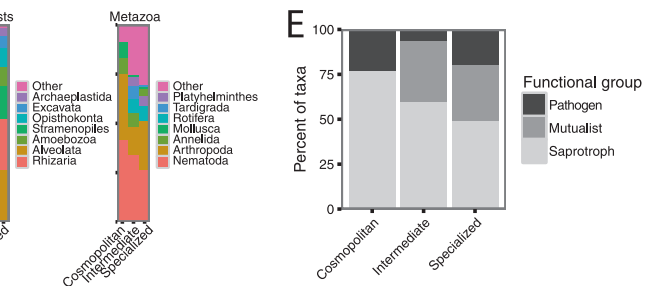

Fig. 1 The effects of plant species identity on the composition of soil communities from mesocosms containing monocultures. Boxplots represent pairwise Bray-Curtis dissimilarities in community composition between vs. within soils from the same plant species (a). Hierarchical clustering diagrams based on mean dissimilarities across the plant species (b). Bipartite network diagram, where edges (lines) connect plant species (green circles) to fungal taxa (red points) that

temperate grassland under extensive agricultural management, which involved light grazing by sheep and cattle from autumn to spring, but no grazing during the growing season when an annual hay crop was taken, and an occasional light dressing of farmyard manure or mineral fertilizer $(\sim 25 \mathrm{~kg}$ ha $\left.{ }^{-1} \mathrm{~N}\right)$ in early spring [39]. Twenty-one grassland plant species (Fig. 1) were germinated and grown in a greenhouse from commercial seed (Emorsgate Seeds, Norfolk, PE34 4RT, UK) or from seed collected at the site. Mesocosms were planted and arranged in a randomized block design with four blocks. Plants were actively weeded and harvested annually. Plant biomass and soil was collected in July, approximately two years following planting, during the height of the growing season and before seed filling. Eight to 20 leaves from at least three individuals per mesocosm occurred in the same mesocosm (c). The composition of cosmopolitan soil taxa (those taxa associated with all plant species), intermediate (taxa associated with only 2 to 20 plant species), and specialized (taxa that associate with only a single plant species) (d). The composition of functional groups of fungal taxa identified as being cosmopolitan, intermediate, and specialized across plant species (e)

were clipped and stored in sealed plastic bags at $4{ }^{\circ} \mathrm{C}$ prior to processing. A representative $6.8 \mathrm{~cm}$ diameter soil core was taken from the complete soil column of each mesocosm, and soil subsamples were frozen and shipped on dry ice to the University of Colorado for molecular soil community analysis. The remainder of the soil was immediately passed through a 4-mm sieve. All root material not passing through the sieve was retained and stored at $4{ }^{\circ} \mathrm{C}$ before being washed free of soil prior to processing for root trait measurements.

\section{Field plots design and sampling}

Experimental field plots were established $2 \mathrm{~km}$ from the mesocosm enclosure at Selside Shaw, within the Ingleborough National Nature Reserve. The plots were established in 
2012, in a mesotrophic grassland with similar management, vegetation and soil to the meadow at Colt Park. The soil was characterized as a clayey brown earth soil with $60 \%$ clay, $<1 \%$ silt, $39 \%$ sand, $5.7 \pm 0.4 \mathrm{pH}$ (mean \pm standard deviation), $4.9 \pm 1.4 \% \mathrm{C}$, and $0.46 \pm 0.13 \% \mathrm{~N}$. Native grassland species were added to the existing plant communities in 6 $\mathrm{m} \times 6 \mathrm{~m}$ field plots with the aim of creating a gradient of plant communities of increasing functional diversity and complexity. Over two years the plots were seeded (2014-2015) and planted with seedlings (2013-2015) of species belonging to one of three plant functional groups, namely the grasses (Cynosurus cristatus, Dactylis glomerata, Festuca rubra, Poa trivialis, and Briza media), forbs (Achillea millefolium, Geranium sylvaticum, Geum rivale, Leucanthemum vulgare, Plantago lanceolata, Prunella vulgaris, Hypochaeris radicata, Leontodon hispidus, Filipendula ulmaria, and Centaurea nigra), and legumes (Lathyrus pratensis, Lotus corniculatus, Trifolium pretense, and Trifolium repens) or their respective two- and three-way combinations. These species are typical of species-rich mesotrohic meadow communities (UK National Vegetation Classification MG3b; [40]), the target plant community for biodiversity [41]. Together with unmodified control communities, this created a total of eight plant community treatments with five replicates of each arranged in a randomized design ( $n=40$ plots). Details on species added, seedling densities, and sowing rates across all treatments are given in Table S1. We note that most, but not all, of the species contained in the mesocosms were represented in the field plots.

We sampled vegetation and soil from four of the eight treatments (control, forb addition, legume addition, and grass-forb-legume addition) in July 2015. To sample vegetation and soil, $30 \mathrm{~cm}$ diameter sampling rings were placed at representative locations within plots $(n=4$ per plot with 5 plots per treatment; i.e. $n=20$ per treatment), and aboveground plant biomass was harvested from within each sampling ring. One $6.8 \mathrm{~cm} \times 10 \mathrm{~cm}$ soil core was collected from within the center of each sampling ring and processed identically to the mesocosm soil samples. Root material was processed as above for use in the root-based assessment of plant community composition.

\section{Soil community composition}

Fungal, bacterial, protistan, and metazoan communities were assessed in soil samples following molecular marker gene sequencing protocols as described in Prober et al. [16] and Ramirez et al. [42]. Briefly, DNA was extracted from each sample, and ribosomal marker genes were amplified using PCR with barcoded primers unique to each sample. We used the ITS1F/ITS2 and the $515 \mathrm{f} / 926 \mathrm{r}$ primer pairs for fungi and bacteria, respectively, and the 1391f/EukBr primer set for protists and metazoa. Amplicon pools were sequenced on an Illumina MiSeq instrument using $2 \times 251$ bp sequencing kits at the BioFrontiers sequencing facility at the University of Colorado. Appropriate controls were used throughout the laboratory process to ensure there were no contaminants. Raw sequence data are available at figshare. com using the following digital object identifiers https://doi. org/10.6084/m9.figshare.4879940, https://doi.org/10.6084/ m9.figshare.4879889, https://doi.org/10.6084/m9.figshare. 4879943.

Raw sequences were processed using the DADA2 pipeline [43], which is designed to resolve exact biological sequences from Illumina sequence data and does not involve sequence clustering. Raw sequences were first demultiplexed by comparing index reads to a key, and paired sequences were trimmed to uniform lengths. Sequences were then dereplicated, and the unique sequence pairs were denoised using the 'dada' function with 'err = NULL' and 'selfConsist = TRUE'. Potential primers and adapters were then screened and removed using a custom script (https://github.com/leffj/da da2helper). Next, paired-end sequences were merged and chimeras were removed. Taxonomy assignments were determined using the RDP classifier trained on the UNITE [44], Greengenes [45], or PR2 databases [46] for fungi, bacteria and protists and metazoa, respectively. Zygomycota classifications were changed to Mucoromycota as per Spatafora et al. [47]. 16S rRNA gene sequences identified as chloroplasts, mitochondria, or Archaea were removed. To account for differences in sequencing depths, samples were rarefied to 5300, 1300, 2400, and 1250 sequences per sample for fungi, bacteria, protists, and metazoa, respectively. Putative fungal functional groups were identified using FUNGuild [48].

\section{Plant community composition}

Plant community composition in the field plot samples was assessed in four ways: (1) by sorting the aboveground biomass to species and measuring the biomass (dry weight) of each species, (2) by molecular analysis of the aboveground biomass, (3) by molecular analysis of the roots contained in the soil cores, and (4) by molecular analysis of DNA extracted from the soil samples. For visual inspection, harvested aboveground biomass was identified the same day as collection, and tissue from each species was dried and weighed. For molecular assessments, aboveground and root biomass samples were freeze-dried, ground, and homogenized prior to DNA extraction. We prepared DNA for sequencing following a protocol similar to Kartzinel et al. [49]. We identified the genus-level plant community composition by targeting both the P6 loop of the trnL gene and the rRNA internal transcribed spacer (ITS) region. We extracted DNA using the PowerSoil DNA Isolation Kit (Mo Bio Laboratories, Inc., Carlsbad, CA, USA), and soil samples were diluted 1:10 prior to amplification. The primer set 
$\operatorname{trnL}(\mathrm{UAA}) \mathrm{c} / \mathrm{trnL}(\mathrm{UAA})$ with included Illumina sequencing adapters was used to amplify the $t r n L-\mathrm{P} 6$ marker following a PCR protocol of: denaturing at $94{ }^{\circ} \mathrm{C}$ for 2 min followed by 36 cycles of $94{ }^{\circ} \mathrm{C}$ for $1 \mathrm{~min}, 55^{\circ} \mathrm{C}$ for $30 \mathrm{~s}$, and $72^{\circ} \mathrm{C}$ for 30 $\mathrm{s}$, with a 5-min final extension at $72^{\circ} \mathrm{C}$. To amplify the ITS region, we used the forward primer, ITS1-F, and included two reverse primers, ITS1Ast-R and ITS1Poa-R [49], to specifically target Asteraceae and Poaceae species. All primers included appropriate Illumina adapters, and PCR reactions were carried out as for trnL amplification. Each PCR was done in duplicate and the amplification product was combined. All products for each sample were combined in equal volumes and cleaned using the UltraClean PCR CleanUp Kit (Mo Bio Laboratories, Inc.). Illumina Nextera barcodes were added to the amplicons using an 8-cycle PCR, amplicons were cleaned and pooled using the SequalPrep kit (Invitrogen, Carlsbad, CA, USA), and sequenced on an Illumina MiSeq instrument with a $2 \times 151 \mathrm{bp}$ kit at the University of Colorado BioFrontiers sequencing facility.

We processed raw plant sequences in a similar manner as for soil community sequences described above. We used the DADA2 pipeline [43] to trim forward and reverse paired reads to 145 and $130 \mathrm{bp}$, respectively. Following the denoising step, Illumina adapters were removed, paired, end reads were merged, and chimeras were filtered. We assigned taxonomy to each sequence using BLAST searches against the GenBank NR database. Sequences were assigned taxonomy only if $\geq 80 \%$ of the sequence aligned to a reference sequence and they matched the reference sequence with $\geq 95 \%$ identity. If a sequence had multiple best matches to reference sequences, a common genus and/or family name was assigned if one existed. Otherwise, sequences were assigned as 'unknown'. Taxonomy assignments were manually checked and verified in reference to species known to exist at the site. Separate taxa tables were created based on trnL amplicons and each of the Asteraceae and Poaceae ITS amplicons. Samples with fewer than 550, 1000, and 100 sequences were removed from taxa tables based on trnL, Asteraceae ITS, and Poaceae ITS amplicons, respectively. We calculated the relative abundance of individual plant genera in each sample using the trnL sequence counts. Because the trnL gene yields limited taxonomic resolution for the Asteraceae and Poaceae, we replaced the total relative abundances of taxa (mostly unknown genera) within these two families with normalized relative abundances of genera determined using the ITS sequence data. Raw sequence data are available at figshare. com using the https://doi.org/10.6084/m9.figshare.4880060.

\section{Plant traits}

All leaf and root traits were measured using standard protocols [50]. Briefly, we measured specific leaf area, specific root length, leaf dry matter content, and root dry matter content by weighing and scanning the fresh leaf and root samples. The samples were then oven dried at $60{ }^{\circ} \mathrm{C}$ for 48 $\mathrm{h}$ and their dry weights measured. The scanned digital images were analyzed in WinRhizo (Reagent Instruments Inc., Ville de Québec, QC, Canada) to determine leaf areas, root lengths, and root diameters. Shoot and root $\mathrm{N}$ and $\mathrm{C}$ contents from the mesocosm-grown plants and the field sample plant communities were measured on an Elementar Vario elemental analyzer (Langenselbold, Germany). In both cases, plant material was freeze-dried and thoroughly homogenized prior to measurement.

\section{Soil characteristics}

Soil characteristics were measured as in Orwin et al. [35]. $\mathrm{pH}$ was measured using a ratio of $1 \mathrm{~g}$ fresh soil: $2.5 \mathrm{ml}$ $\mathrm{dH}_{2} \mathrm{O}$. Dissolved inorganic $\mathrm{N}$, individual ions $\left(\mathrm{NO}_{3}-\mathrm{N}\right.$, $\mathrm{NH}_{4}-\mathrm{N}$ ), and net $\mathrm{N}$ mineralization were assessed using $1 \mathrm{M}$ $\mathrm{KCl}$ extracts, and dissolved organic $\mathrm{N}$ was assessed using water extracts as in Bardgett et al. [51]. Total soluble $\mathrm{N}$ was determined following oxidation of these extracts using potassium persulphate [51]. Extracted mineral fractions were quantified using standard spectrophotometric protocols on a AA3 segmented flow analyser (SEAL Analytical Inc., Mequon, WI, USA). Total $\mathrm{C}$ and $\mathrm{N}$ of dried and ground subsamples were measured using an Elementar Vario EL elemental analyzer.

\section{Statistical analyses}

All statistical analyses were performed in R [52] using specific packages where noted, and the package 'mctoolsr' (http://leffj.github.io/mctoolsr/) was used to facilitate data manipulation and analyses. To represent differences in community composition, we calculated Bray-Curtis dissimilarities using square-root transformed relative abundances. Permutational analysis of variance (PERMANOVA), as implemented in the 'adonis' function from the 'vegan' package, was used to test for differences in soil community composition across factors. To test for differences in soil community composition across mesocosm plant species, we used PERMANOVA and included block identity as a random factor in the model. Network analysis plots were created using the 'igraph' package with multidimensional scaling to distribute points. Soil taxa were considered present if their mean relative abundance was $\geq 0.1 \%$, and only taxa with a relative abundance $>0.5 \%$ that associated with $\geq 1$ plant species are shown. We identified particular soil taxa that associated with specific plant species using indicator analyses [53]. 'Cosmopolitan' soil taxa were defined as those taxa associated with all plant species (i.e. had a mean relative abundance $\geq 0.1 \%$ across replicates for each 
species), 'intermediate' as taxa associated with only 2 to 20 plant species, and 'specialized' as taxa that associated with only a single plant species.

To test the relationship between the composition of soil communities and plant species relatedness in the mesocosms, we used the phylogeny from Durka and Michalski [54]. Relationships between difference in soil community composition and plant phylogenetic distances were evaluated using Mantel tests with Spearman correlations. We tested for a phylogenetic signal in the relative abundance of individual protist taxa using the phylosig function in the 'phytools' package, where the statistic, $K$, represents the strength of the signal [55]. We calculated multivariate dissimilarities in trait values by normalizing and standardizing individual trait values and calculating Euclidian distances. We tested the relationship between Euclidian trait distances and community composition dissimilarities using Mantel tests.

For the field samples, we calculated differences in the phylogenetic structure of plant communities (i.e. phylogenetic dissimilarity) using UniFrac [56] as implemented in the package, 'picante'. We used the plant phylogenetic tree as reported in Durka and Michalski [54], and plants not identified to the genus level were removed. We assessed the relationship between phylogenetic dissimilarity and the Bray-Curtis dissimilarities in soil community composition using Mantel tests with Spearman correlations.

To assess whether differences in plant community composition predicted variation in soil community composition beyond the explanatory power of soil characteristics, we built models of soil community composition dissimilarity using multiple regression on distance matrices (MRM) as implemented in the 'ecodist' package and compared the explanatory power of the model with and without the addition of plant community dissimilarity as a predictor variable. In these models, each soil variable was transformed using log or inverse transformations where necessary to approximate a normal distribution, and they were standardized prior to calculating Euclidian distances. MRM was implemented with rank (i.e. Spearman) correlations, and the "best" models containing only soil variables were derived by first including all soil variables and using backwards elimination until all predictors explained significant levels of variation in the response dissimilarities.

\section{Results and discussion}

\section{The effect of plant species identity on soil communities}

Overall, the mesocosm soils contained expectedly diverse communities (Fig. S1A). Soil fungal communities were primarily composed of Ascomycota [43\% of ITS sequence reads, on average], Basidiomycota (31\%), and Mucoromycota (21\%); bacterial communities were primarily composed of Acidobacteria (31\% of 16S rRNA gene reads, on average), Proteobacteria (20\%), and Verrucomicrobia $(16 \%)$; protistan communities were primarily composed of Rhizaria (26\%), Amoebozoa (25\%), Alveolata (22\%), and Stramenopiles (16\%); and metazoan communities were primarily composed of Nematoda (33\%), Arthropoda (28\%), and Annelida (15\%; Fig. S1B). The structure of these communities was similar to those found in other temperate grasslands [1, 57, 58].

Plant species identity explained differences in the overall composition of soil fungal $\left(R^{2}=0.33 ; P<0.001\right)$, bacterial $\left(R^{2}=0.27 ; P=0.02\right)$, protistan $\left(R^{2}=0.32 ; P<\right.$ $0.001)$, and metazoan $\left(R^{2}=0.31 ; P<0.001\right)$ communities (Fig. 1a). Further, these plant species effects were driven by differences among multiple plant species rather than one or a small number of plant species associating with distinct belowground communities (Fig. 1b, Fig. S2). Certain fungal, protistan, and metazoan taxa tended to be strongly associated with individual plant species, while others tended to have more general associations (Fig. 1c, Fig. S3). For example, the fungal taxa identified as Olpidium brassicae and Phoma sp. associated with Achillea millefolium, while several Ascomycota, Basidiomycota, and Mucoromycota taxa were associated with all plant species (Fig. S4). We used an indicator analysis approach to identify those taxonomic groups that were most strongly associated with each of the individual plant species and found that many of the plant species formed specific associations (Fig. S4). Since there are likely to be different traits associated with more specialized versus more cosmopolitan soil taxa [59], we investigated whether soil taxa unique to individual plant species tended to represent different taxonomic groups when compared to taxa that were more ubiquitous across plant species. Cosmopolitan taxa were represented by a higher proportion of Mucoromycota, Acidobacteria, Rhizaria, and Nematoda, while more specialized taxa were represented by a greater proportion of Glomeromycota, Planctomycetes, Alveolata, and Rotifera (Fig. 1d). Additionally, cosmopolitan fungal taxa represented a greater proportion of putative saprotrophs compared to more specialized taxa, which had a greater proportion of pathogens and mutualists (Fig. 1e). This suggests that, in temperate grasslands, pathogens and mutualists tend to be more strongly limited to individual plant species, while saprotrophs are more cosmopolitan and less influenced by plant species identity. This finding is in concordance with a previous study conducted in an Amazon rainforest showing stronger plant-soil linkages for pathogenic and mycorrhizal fungi compared to saprotrophs [60]. 


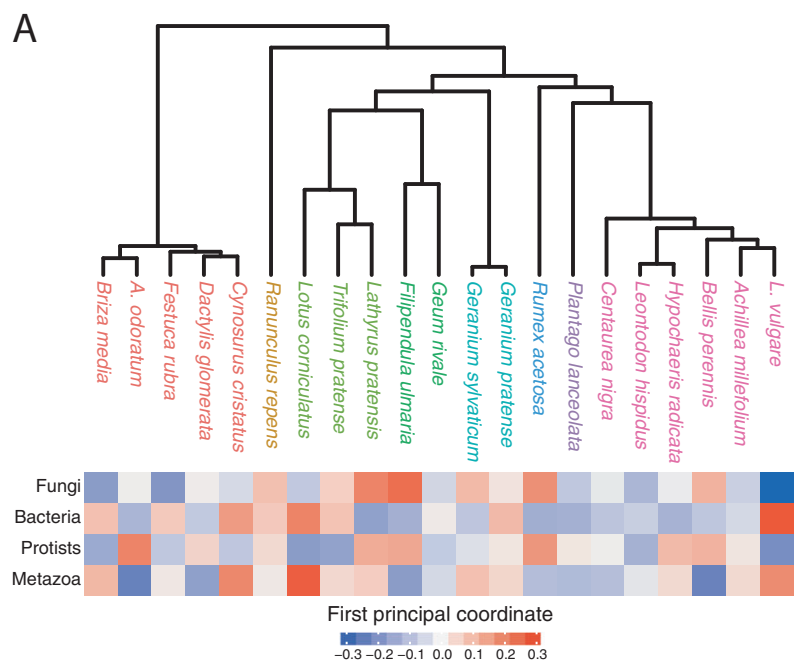

Fig. 2 Relationships between plant species' relatedness and differences in the composition of soil communities. Panel a shows a plant phylogenetic tree with species names colored by family (key shown in Fig. 1) with the corresponding heatmap showing the dissimilarities in the composition of each soil community. Colors represent the first principal coordinate analysis axis calculated from Bray-Curtis

\section{Can the effect of plant species identity be explained by plant phylogeny or functional traits?}

We next sought to assess whether plant species identity effects could be explained by plant phylogeny or leaf and root functional traits, two attributes that could potentially be used to predict plant associations with belowground communities a priori. The mesocosm plant species represented eight families including Poaceae, Asteraceae, and Fabaceae, providing an opportunity to evaluate the influence of a wide-ranging phylogeny on the composition of soil communities. Plant phylogenetic distances were not significantly related to differences in fungal, bacterial, or metazoan community composition $(P>0.1$ in all cases; Fig. 2a). Differences in protistan community composition were related to plant phylogenetic distance, but this relationship was relatively weak (rho $=0.29, \quad P=0.002$; Fig. 2a). Nonetheless, the relative abundance of Stramenopiles was significantly related to plant species phylogeny ( $K=0.51, P=0.004$; Fig. S5). We might expect plant phylogenetic differences to be associated with the structure of belowground communities due to coevolution with mutualists or pathogens [28, 61]; however, this did not appear to be the case for most soil taxonomic groups. Further, the general lack of a relationship between plant phylogeny and belowground communities found in our study is consistent with studies of plant-soil feedbacks, which likewise have shown no relation to plant phylogeny [62].

The measured leaf and root traits were highly variable across the mesocosm species. Grassland plants vary in their
B

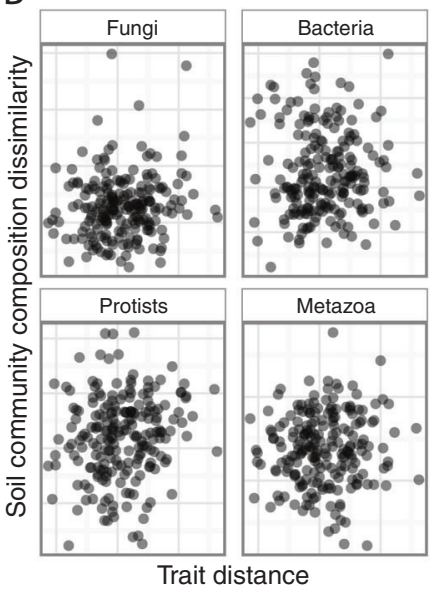

C

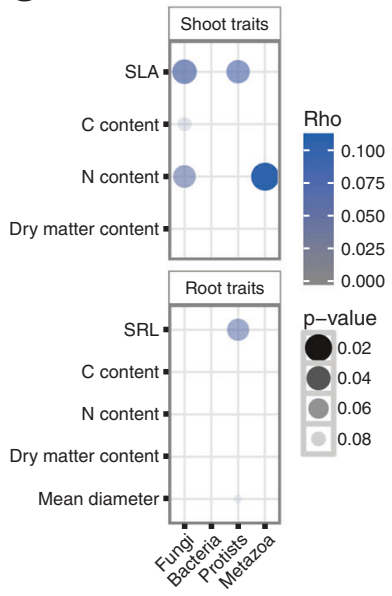

dissimilarities (a). The relationship between differences in the composition of soil communities and plant trait distances (b). Euclidean trait distances were calculated using all the traits shown in panel c. The relationship between differences in the composition of soil communities and individual plant traits (c). Points represent Spearman correlation coefficients (rho) and Mantel test results ( $P$-value)

ecological strategies. Exploitative species grow fast under high nutrient conditions and have characteristically high specific leaf areas and $\mathrm{N}$ contents while conservative species are selected to survive under lower nutrient conditions and have opposite traits [63, 64]. For each plant species in the mesocosms, we measured the plant traits that are known to be indicative of the tradeoffs in these life history strategies (Fig. S6A, Table S2). For example, the Fabaceae species tended to have a greater shoot and root $\mathrm{N}$ and $\mathrm{C}$ content, while Poaceae species tended to have high leaf dry matter contents (Fig. S6B). Yet, there were no strong or significant relationships (i.e. Bonferroni corrected $P<0.05$ ) between belowground community composition and individual leaf or root traits (Fig. 2c). Furthermore, multivariate dissimilarity in leaf and root traits of plant species was not predictive of differences in communities of any of the soil taxonomic groups $(P>0.1$ in all cases; Fig. $2 b)$.

These results suggest that the plant traits we measured are not effective indicators of the specific relationships plants form with belowground communities. Previous studies have detected relationships between plant traits and coarse measures of microbial community composition [15, 35] or specific microbial groups, such as ammonia oxidizers [37]. However, our findings are in line with other studies. For example, Porazinska et al. [25] found that certain soil communities were linked to individual plant species in a prairie grassland, but they were unable to identify traits that could predict soil communities. Likewise, Barberán et al. [65] demonstrated that plant species identity is more predictive of soil communities than plant traits. Nonetheless, it is possible that the plant-soil organism associations we 
observed could have been driven by unmeasured plant traits given that certain plant characteristics must explain the species identity effects we observed. For example, variations in the quantity and quality of root exudates can influence soil community composition [66]. Likewise, leaf litter chemistry has been shown to be related to coarse measures of soil microbial community composition in a manner broadly consistent with the leaf economic spectrum [35]. Also, while we did not observe relationships between plant traits and the overall composition of soil communities, it is possible that specific soil organisms do respond to plant traits, including those taxa directly involved with $\mathrm{N}$ cycling $[34,36,37]$. Other potential reasons exist for our failure to detect strong associations between soil communities and plant traits or phylogeny. First, it is possible that if the experiment had a longer duration, additional effects on soil communities would become evident, and these effects would more strongly correspond to differences in plant traits and/or phylogeny. Second, soil can contain DNA from cells that are no longer viable [67], and this 'relic' DNA could obscure ecological relationships among organisms.

\section{Are soil communities in the field predictable based on plant community attributes?}

The results from the mesocosm study demonstrated that plant species identity is a more important determinant of soil community composition than plant phylogeny or plant traits. Given this, we would hypothesize that knowledge of the species composition of mixed plant communities in the field should be an effective predictor of soil communities. We tested this hypothesis by analyzing plant and soil samples from a series of experimental plots established at a grassland site close to the mesocosm experiment, where grassland community composition had been manipulated for three years to create a gradient of plant species composition and diversity. Plant community composition was assessed using marker gene sequencing of plant DNA extracted from dried and ground representative samples of plant biomass collected immediately above each soil sample, and this molecular approach was verified for efficacy by comparing it to visual assessments of aboveground biomass (Fig. S7).

Differences in the composition of each soil taxonomic group were related to differences in plant community composition $(P<0.05$ in all cases). By comparing the compositions of the plant communities across experimental plots (using the first principal coordinate score based on aboveground assessments), we could identify specific plant genera that drove variation in soil community composition across the samples (Fig. 3a, Table S3). For instance, some samples had comparatively high relative abundances of Lolium spp. while other samples had high relative abundances of Agrostis spp. These differences in plant community composition were related to the relative abundance of certain groups of soil taxa, including the Ascomycota, Mucoromycota, Acidobacteria, Amoebozoa, Stramenopiles, and Arthropoda (Fig. 3a). These specific associations between plant and soil taxa can ultimately be used to predict the composition of soil communities from plant species abundances. For example, our results suggest that plant communities dominated by Agrostis spp. are likely to have greater relative abundances of Ascomycota and lower relative abundances of Acidobacteria in the soils in which they grow.

We also evaluated whether the phylogenetic structure or community-aggregated plant traits [15, 32] could explain relationships between plants and soil communities. We did this by testing whether plant communities containing genera with more similar phylogenetic histories or trait values were associated with more similar soil communities. However, plant community phylogenetic structure was not significantly related to the composition of any of the soil taxonomic groups $(P>$ 0.3 in all cases), suggesting that phylogenetic relatedness is not predictive of soil community composition. This finding is in agreement with the monoculture mesocosm study described above and a field study conducted in a tropical rainforest that failed to find a strong effect of tree species phylogenetic relationships on soil communities [27]. Furthermore, differences in community-aggregated trait values, including leaf and root $\mathrm{N}$ and $\mathrm{C}$ content, also did not significantly relate to the composition of any of the soil taxonomic groups $(P>0.1$ in all cases). The trait values we measured were not predictive of soil community composition in mixed grassland communities, results that are consistent with those from the mesocosm experiment of individual plant species.

In addition to assessing relationships between the composition of soil taxonomic groups and plant communities based on aboveground biomass, we evaluated plant community composition in two other ways: using root DNA and plant DNA in soil. We used these approaches because roots of different species are intermingled and difficult to identify visually, and assessing plant communities via soil DNA provides an alternate approach to determine which plant species have occupied a given location currently or in the past [68]. Roots might also might be more strongly associated with soil community structure than aboveground tissue [35]. As with the aboveground plant biomass-based analysis, differences in the compositions of each of the soil taxonomic groups were related to differences in plant community composition assessed using the plant DNA extracted from soil $(P<0.05$ in all cases). However, the differences in the composition of soil communities were not significantly related to differences in plant community composition assessed using root DNA $(P>0.1$ in all cases; Fig. 3b). It is possible that the composition of plant communities as assessed via roots were unrelated to soil 


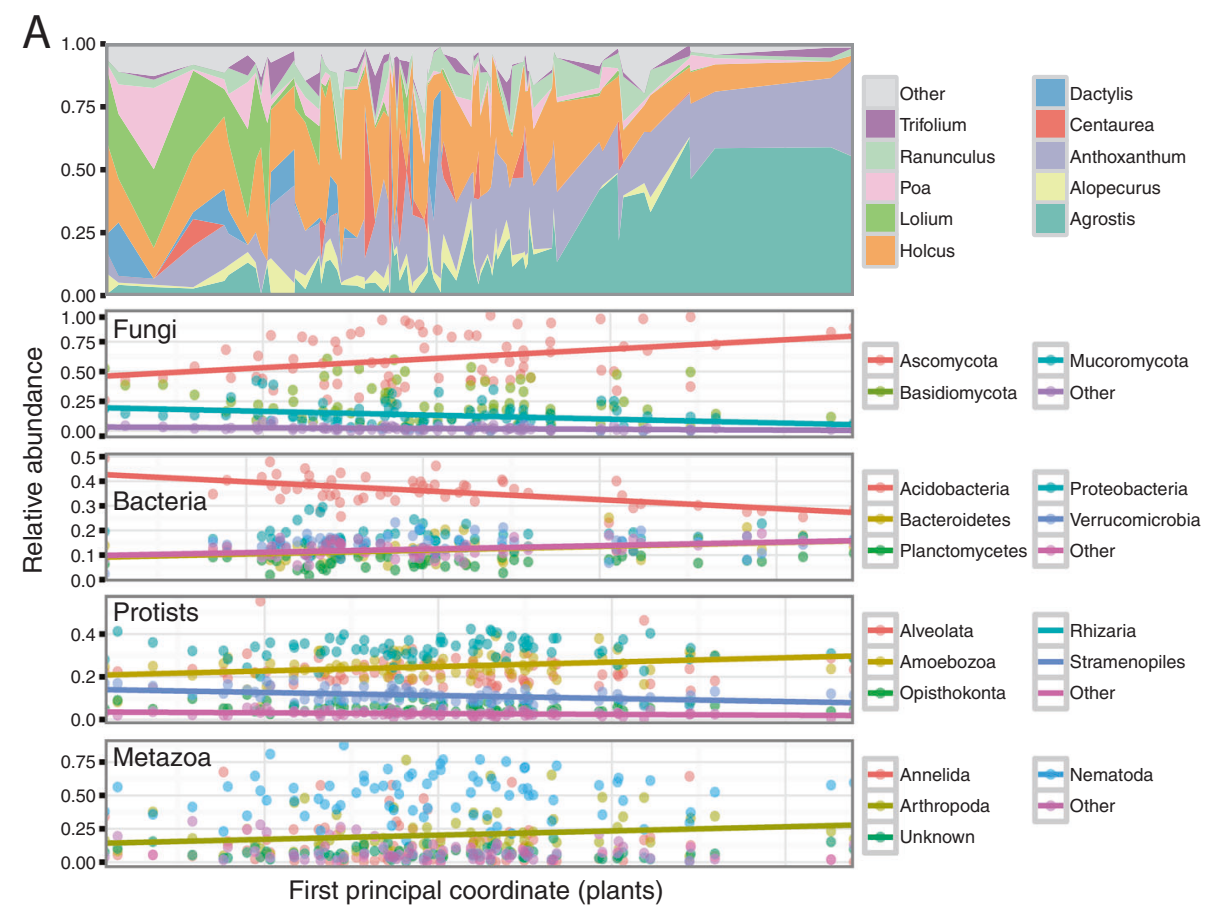

B
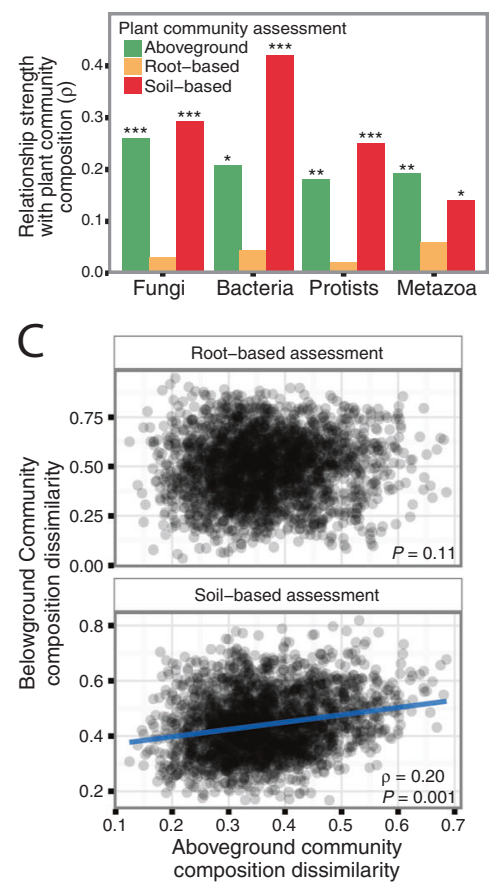

Fig. 3 Soil community composition is related to plant community composition in the field. Variation in plant community composition across the field samples ordered by the first principal coordinate score (i.e. the $x$-axis represents a gradient of plant community compositions where communities further apart are more dissimilar), and relationships between soil taxonomic group relative abundance and the plant first principal coordinate score (a). Linear trend lines were only plotted for groups that had a Pearson correlation $P \leq 0.05$. Relationship

communities because much of the root biomass consisted of dormant plants or dead tissue [69]. Further, it is possible that root distributions are so variable over time that they obscure plant species effects on belowground communities.

Differences in aboveground plant community composition were unrelated to differences in root community composition $(P=0.11)$, but they were related to differences in the plant community composition as assessed using plant DNA in soil (rho $=0.2 ; P<0.001 ;$ Fig. $3 \mathrm{c}$ ). This shows that shoot and root biomass in a given location do not represent the same plant community, as also found in a tropical rainforest [27]. Additionally, these results suggest that plant DNA in soil can be used as a proxy for the community composition of the aboveground biomass [68]. This has implications for future research since it is often logistically easier to obtain a representative sample of surface soils rather than sampling and homogenizing aboveground plant biomass.

\section{Are the associations between plant and soil communities driven by soil characteristics?}

We aimed to assess whether relationships between soil communities and plant communities in the field plots were attributable to the direct effects of the plants, shared strength between dissimilarities in soil communities and dissimilarities in plant communities $(* P<0.05, * * P<0.01, * * * P=0.001$; Mantel tests; b). Pairwise Bray-Curtis dissimilarities in plant community composition, as assessed using aboveground tissue, are not related to dissimilarities in plant community composition as assessed using root tissue, but they are related to dissimilarities in plant community composition as assessed using plant DNA in soil (c)

environmental drivers, or intermediary effects of the plants on soil properties. Therefore, we evaluated whether plant community composition contributed additional explanatory power to the observed variation in soil community composition given differences in edaphic characteristics. Shifts in the composition of soil communities across the field plots were significantly correlated with multiple, individual edaphic properties (Table S4), and combinations of these properties explained 13-29\% of the variation in soil community composition ( $P=0.001$ in all cases; Fig. S8A). For example, soil $\mathrm{N}$ content and $\mathrm{pH}$ were typically predictive of the composition of the four taxonomic soil groups. Only differences in fungal community composition could be predicted more accurately when information on aboveground plant community composition was added to the models containing only soil characteristics as predictor variables $(P=0.01$; Fig. S8). When soil DNA-based plant community composition information was used instead of aboveground plant community composition, fungal, bacterial, and protistan community composition could all be predicted more accurately with the addition of information on plant community composition $\left(R^{2}\right.$ increased $9-24 \% ; P<$ 0.02 in all cases; Fig. S8). These results suggest that shifts in aboveground community composition likely influence 
soil communities in ways not accounted for in commonly measured soil properties, and indicate that the structure of complex soil communities in grasslands is controlled by a combination of plant and soil characteristics $[11,70]$.

\section{Conclusions}

We demonstrate that plant community composition is an effective predictor of the structure of complex grassland soil communities, especially when combined with information on soil abiotic properties. Furthermore, we show that plant community composition is particularly effective for predicting distributions of certain groups of soil organisms, such as fungal symbionts and pathogens. Importantly, we found that plant species identity, rather than plant phylogeny or functional traits, was the best predictor of soil community composition at both the individual plant and community scale. This is significant because it raises questions about the effectiveness of phylogenetic and traitbased approaches for explaining spatial variation in soil community composition at a local scale. Such approaches are increasingly being used to predict how changes in plant community composition impact soil properties and functions $[38,71]$, but our findings indicate that, at a local scale in temperate grasslands, they are ineffective for explaining variation in soil communities. Finally, it is important to note that much of the variation in soil community composition could not be explained by the measured soil characteristics or plant community attributes, highlighting the difficulty of predicting complex soil communities in situ and the need to build a mechanistic understanding of which specific plant attributes are responsible for driving plant species effects on the biodiversity of soil. Combined, our findings provide new evidence that associations between specific plant species and complex soil communities, associations that are not explained by plant phylogeny or commonly measured plant traits, act as key determinants of spatial patterns of biodiversity in grassland soils.

\footnotetext{
Acknowledgements We thank Emily Morgan for her assistance with the microbial community analyses, Colin Newlands of Natural England for permission to use the field sites, and Marina Semchenko for comments on a previous version of this manuscript. We also thank all who helped establish the field experimental site and collect and process the plant and soil samples: Aurore Kaisermann, Debora Ashworth, Angela Straathof, Imelda Uwase, Marina Semchenko, Maatren Schrama, Melanie Edgar, Mark Bradford, Mike Whitfield, Rachel Marshall, and Andrew Cole.
}

Funding This research was supported by a grant from the UK Biotechnology and Biological Sciences Research Council (BBSRC) (Grant BB/I009000/2), initiated and led by RDB, a BBSRC International Exchange Grant (BB/L026406/1) between RDB and NF, and a grant from the U.S. National Science Foundation (NSF) (DEB 1542653) awarded to NF.

\section{Compliance with ethical standards}

Conflict of interest The authors declare that they have no conflict of interest.

\section{References}

1. Bates ST, Clemente JC, Flores GE, Walters WA, Parfrey LW, Knight R, et al. Global biogeography of highly diverse protistan communities in soil. ISME J. 2013;7:652-9.

2. Fierer N, Strickland MS, Liptzin D, Bradford Ma, Cleveland CC. Global patterns in belowground communities. Ecol Lett. 2009;12:1238-49.

3. Kaiser K, Wemheuer B, Korolkow V, Wemheuer F, Nacke H, Schöning I, et al. Driving forces of soil bacterial community structure, diversity, and function in temperate grasslands and forests. Sci Rep. 2016;6:33696.

4. Tedersoo L, Bahram M, Polme S, Koljalg U, Yorou NS, Wijesundera R. et al. Global diversity and geography of soil fungi. Science. 2014;346:1256688.

5. Van der Putten WH, Bardgett RD, Bever JD, Bezemer TM, Casper BB, Fukami T, et al. Plant-soil feedbacks: the past, the present and future challenges. J Ecol. 2013;101:265-76.

6. Wardle DA, Bardgett RD, Klironomos JN, Setälä $\mathrm{H}$, van der Putten WH, Wall DH. Ecological linkages between aboveground and belowground biota. Science. 2004;304:1629-33.

7. Bardgett RD, Wardle DA. Aboveground-belowground linkages: biotic interactions, ecosystem processes, and global change. New York, NY, USA: Oxford University Press Oxford; 2010.

8. van der Heijden MGA, Klironomos JN, Ursic M, Moutoglis P, Streitwolf-Engel R, Boller T, et al. Mycorrhizal fungal diversity determines plant biodiversity, ecosystem variability and productivity. Nature. 1998;396:69-72.

9. Hiiesalu I, Pärtel M, Davison J, Gerhold P, Metsis M, Moora M, et al. Species richness of arbuscular mycorrhizal fungi: associations with grassland plant richness and biomass. New Phytol. 2014;203:233-44.

10. Singh BK, Millard P, Whiteley AS, Murrell JC. Unravelling rhizosphere-microbial interactions: opportunities and limitations. Trends Microbiol. 2004;12:386-93.

11. Berg G, Smalla K. Plant species and soil type cooperatively shape the structure and function of microbial communities in the rhizosphere. FEMS Microbiol Ecol. 2009;68:1-13.

12. Grayston SJ, Wang S, Campbell CD, Edwards AC. Selective influence of plant species on microbial diversity in the rhizosphere. Soil Biol Biochem. 1998;30:369-78.

13. Bardgett RD, Mawdsley JL, Edwards S, Hobbs PJ, Rodwell JS, Davies WJ. Plant species and nitrogen effects on soil biological properties of temperate upland grasslands. Funct Ecol. 1999;13:650-60.

14. Grayston SJ, Griffith GS, Mawdsley JL, Campbell CD, Bardgett RD. Accounting for variability in soil microbial communities of temperate upland grassland ecosystems. Soil Biol Biochem. 2001;33:533-51.

15. de Vries FT, Manning P, Tallowin JRB, Mortimer SR, Pilgrim ES, Harrison KA, et al. Abiotic drivers and plant traits explain landscape-scale patterns in soil microbial communities. Ecol Lett. 2012;15:1230-9.

16. Prober SM, Leff JW, Bates ST, Borer ET, Firn J, Harpole WS, et al. Plant diversity predicts beta but not alpha diversity of soil microbes across grasslands worldwide. Ecol Lett. 2015;18:85-95.

17. Lekberg Y, Waller LP. What drives differences in arbuscular mycorrhizal fungal communities among plant species? Fungal Ecol. 2006;24:135-138. 
18. Berg G. Plant-microbe interactions promoting plant growth and health: perspectives for controlled use of microorganisms in agriculture. Appl Microbiol Biotechnol. 2009;84:11-18.

19. Bezemer TM, Fountain MT, Barea JM, Christensen S, Dekker SC, Duyts $\mathrm{H}$, et al. Divergent composition but similar function of soil food webs beneath individual plants: plant species and community effects. Ecology. 2010;91:3027-36.

20. St. John MG, Wall DH, Behan-Pelletier VM. Does plant species co-occurrence influence soil mite diversity? Ecology. 2006;87:625-33.

21. Gibbons SM, Lekberg Y, Mummey DL, Sangwan N, Ramsey $\mathrm{PW}$, Gilbert JA. Invasive plants rapidly reshape soil properties in a grassland ecosystem. mSystems. 2017;2:e00178-16.

22. Hawkes CV, Wren IF, Herman DJ, Firestone MK. Plant invasion alters nitrogen cycling by modifying the soil nitrifying community. Ecol Lett. 2005;8:976-85.

23. Bezemer TM, Lawson CS, Hedlund K, Edwards AR, Brook AJ, Igual JM, et al. Plant species and functional group effects on abiotic and microbial soil properties and plant-soil feedback responses in two grasslands. J Ecol. 2006;94:893-904.

24. Carey CJ, Michael Beman J, Eviner VT, Malmstrom CM, Hart SC. Soil microbial community structure is unaltered by plant invasion, vegetation clipping, and nitrogen fertilization in experimental semi-arid grasslands. Front Microbiol. 2015; 6. https://doi.org/10.3389/fmicb.2015.00466.

25. Porazinska DL, Bardgett RD, Blaauw MB, Hunt HW, Parsons AN, Seastedt TR, et al. Relationships at the abovegroundbelowground interface: plants, soil biota, and soil processes. Ecol Monogr. 2003;73:377-95.

26. Tedersoo L, Bahram M, Cajthaml T, Põlme S, Hiiesalu I, Anslan $\mathrm{S}$ et al. Tree diversity and species identity effects on soil fungi, protists and animals are context dependent. ISME J. 2016;10:346-362.

27. Barberán A, Mcguire KL, Wolf JA, Jones FA, Wright SJ, Turner $\mathrm{BL}$, et al. Relating belowground microbial composition to the taxonomic, phylogenetic, and functional trait distributions of trees in a tropical forest. Ecol Lett. 2015;18:1397-405.

28. De Deyn GB, Van Der Putten WH. Linking aboveground and belowground diversity. Trends Ecol Evol. 2005;20:625-33.

29. Ben-Hur E, Fragman-Sapir O, Hadas R, Singer A, Kadmon R. Functional trade-offs increase species diversity in experimental plant communities. Ecol Lett. 2012;15:1276-82.

30. Adler PB, Fajardo A, Kleinhesselink AR, Kraft NJB. Trait-based tests of coexistence mechanisms. Ecol Lett. 2013;16:1294-306.

31. Cantarel AAM, Pommier T, Desclos-Theveniau M, Diquélou S, Dumont $\mathrm{M}$, Grassein $\mathrm{F}$, et al. Using plant traits to explain plant-microbe relationships involved in nitrogen acquisition. Ecology. 2015;96:788-99.

32. Grigulis K, Lavorel S, Krainer U, Legay N, Baxendale C, Dumont $\mathrm{M}$, et al. Relative contributions of plant traits and soil microbial properties to mountain grassland ecosystem services. J Ecol. 2013;101:47-57.

33. Legay N, Lavorel S, Baxendale C, Krainer U, Bahn M, Binet M$\mathrm{N}$, et al. Influence of plant traits, soil microbial properties, and abiotic parameters on nitrogen turnover of grassland ecosystems. Ecosphere. 2016;7:1-17.

34. Moreau D, Pivato B, Bru D, Busset H, Deau F, Faivre C, et al. Plant traits related to nitrogen uptake influence plant-microbe competition. Ecology. 2015;96:2300-10.

35. Orwin KH, Buckland SM, Johnson D, Turner BL, Smart S, Oakley S, et al. Linkages of plant traits to soil properties and the functioning of temperate grassland. J Ecol. 2010;98:1074-83.

36. Legay N, Baxendale C, Grigulis K, Krainer U, Kastl E, Schloter $\mathrm{M}$, et al. Contribution of above- and below-ground plant traits to the structure and function of grassland soil microbial communities. Ann Bot. 2014;114:1011-21.
37. Thion CE, Poirel JD, Cornulier T, De Vries FT, Bardgett RD, Prosser JI. Plant nitrogen-use strategy as a driver of rhizosphere archaeal and bacterial ammonia oxidiser abundance. FEMS Microbiol Ecol. 2016;92. https://doi.org/10.1093/femsec/fiw091.

38. Bardgett RD, Mommer L, De Vries FT. Going underground: root traits as drivers of ecosystem processes. Trends Ecol Evol. 2014;29:692-9.

39. De Deyn GB, Shiel RS, Ostle NJ, Mcnamara NP, Oakley S, Young I, et al. Additional carbon sequestration benefits of grassland diversity restoration. J Appl Ecol. 2011;48: 600-8.

40. Rodwell JS. (1992). British plant communities. Volume 3. Grassland and montane communities. 3rd ed. Cambridge University Press: Cambridge, U.K.

41. Smith RS, Shiel RS, Bardgett RD, Millward D, Corkhill P, Rolph $\mathrm{G}$, et al. Soil microbial community, fertility, vegetation and diversity as targets in the restoration management of a meadow grassland. J Appl Ecol. 2003;40:51-64.

42. Ramirez KS, Leff JW, Barberán A, Bates ST, Betley J, Crowther TW, et al. Biogeographic patterns in below-ground diversity in New York City's Central Park are similar to those observed globally. Proc R Soc B Biol Sci. 2014;281:1-9.

43. Callahan BJ, McMurdie PJ, Rosen MJ, Han AW, Johnson AJ, Holmes SP. DADA2: high resolution sample inference from amplicon data. Nat Methods. 2016;13:581-3.

44. Abarenkov K, Henrik Nilsson R, Larsson K-H, Alexander IJ, Eberhardt U, Erland S, et al. The UNITE database for molecular identification of fungi-recent updates and future perspectives. New Phytol. 2010;186:281-5.

45. McDonald D, Price MN, Goodrich J, Nawrocki EP, DeSantis TZ, Probst A, et al. An improved Greengenes taxonomy with explicit ranks for ecological and evolutionary analyses of bacteria and archaea. ISME J. 2012;6:610-8.

46. Guillou L, Bachar D, Audic S, Bass D, Berney C, Bittner L, et al. The Protist Ribosomal Reference database (PR2): a catalog of unicellular eukaryote Small Sub-Unit rRNA sequences with curated taxonomy. Nucleic Acids Res. 2013;41: 597-604.

47. Spatafora JW, Chang Y, Benny GL, Lazarus K, Smith ME, Berbee ML, et al. A phylum-level phylogenetic classification of zygomycete fungi based on genome-scale data. Mycologia. 2016;108:1028-46.

48. Nguyen NH, Song Z, Bates ST, Branco S, Tedersoo L, Menke J, et al. FUNGuild: an open annotation tool for parsing fungal community datasets by ecological guild. Fungal Ecol. $2015 ; 20: 241-8$.

49. Kartzinel TR, Chen Pa, Coverdale TC, Erickson DL, Kress WJ, Kuzmina ML, et al. DNA metabarcoding illuminates dietary niche partitioning by African large herbivores. Proc Natl Acad Sci USA. 2015;112:8019-24.

50. Cornelissen JHC, Lavorel S, Garnier E, Díaz S, Buchmann N, Gurvich DE, et al. A handbook of protocols for standardised and easy measurement of plant functional traits worldwide. Aust J Bot. 2003;51:335-80.

51. Bardgett RD, Streeter TC, Bol R. Soil microbes compete effectively with plants for organic-nitrogen inputs to temperate grasslands. Ecology. 2003;84:1277-87.

52. $\mathrm{R}$ Core Team R: A language and environment for statistical computing 2016. R Foundation for Statistical Computing: Vienna, Austria.

53. Dufrêne M, Legendre P. Species assemblages and indicator species: The need for a flexible asymmetrical approach. Ecol Monogr. 1997;67:345-66.

54. Durka W, Michalski SG. Daphne: a dated phylogeny of a large European flora for phylogenetically informed ecological analyses. Ecology. 2012;93:2297. 
55. Blomberg SP, Garland T, Ives AR. Testing for phylogenetic signal in comparative data: behavioral traits are more labile. Evolution. 2003;57:717-45.

56. Lozupone C, Lladser ME, Knights D, Stombaugh J, Knight R. UniFrac: an effective distance metric for microbial community comparison. ISME J. 2011;5:169-72.

57. Leff JW, Jones SE, Prober SM, Barberan A, Borer ET, Firn JL, et al. Consistent responses of soil microbial communities to elevated nutrient inputs in grasslands across the globe. Proc Natl Acad Sci USA. 2015;112:10967-72.

58. Wu T, Ayres E, Bardgett RD, Wall DH, Garey JR. Molecular study of worldwide distribution and diversity of soil animals. Proc Natl Acad Sci USA. 2011;108:17720-5.

59. Lennon JT, Aanderud ZT, Lehmkuhl BK, Schoolmaster DR. Mapping the niche space of soil microorganisms using taxonomy and traits. Ecology. 2012;93:1867-79.

60. Peay KG, Baraloto C, Fine PVA. Strong coupling of plant and fungal community structure across western Amazonian rainforests. ISME J. 2013;7:1852-61.

61. Anacker BL, Klironomos JN, Maherali H, Reinhart KO, Strauss SY. Phylogenetic conservatism in plant-soil feedback and its implications for plant abundance. Ecol Lett. 2014;17:1613-21.

62. Mehrabi Z, Tuck SL. Relatedness is a poor predictor of negative plant-soil feedbacks. New Phytol. 2015;205:1071-5.

63. Lavorel S, Garnier E. Predicting changes in community composition and ecosystem functioning from plant traits: revisiting the Holy Grail. Funct Ecol. 2002;16:545-56.
64. Roumet C, Birouste M, Picon-Cochard C, Ghestem M, Osman N, Vrignon-Brenas $\mathrm{S}$ et al. Root structure-function relationships in 74 species: evidence of a root economics spectrum related to carbon economy. New Phytol. 2016. https://doi.org/10.1111/nph. 13828.

65. Barberán A, Dunn RR, Reich BJ, Pacifici K, Laber EB, Menninger HL, et al. The ecology of microscopic life in household dust. Proc R Soc B. 2015;282:1-9.

66. Haichar FEZ, Marol C, Berge O, Rangel-Castro JI, Prosser JI, Balesdent $\mathrm{J}$, et al. Plant host habitat and root exudates shape soil bacterial community structure. ISME J. 2008;2:1221-30.

67. Carini P, Marsden PJ, Leff JW, Morgan EE, Strickland MS, Fierer N. Relic DNA is abundant in soil and obscures estimates of soil microbial diversity. Nat Microbiol 2016;2. https://doi.org/10. 1038/nmicrobiol.2016.242.

68. Yoccoz NG, Bråthen KA, Gielly L, Haile J, Edwards ME, Goslar $\mathrm{T}$, et al. DNA from soil mirrors plant taxonomic and growth form diversity. Mol Ecol. 2012;21:3647-55.

69. Hiiesalu I, Öpik M, Metsis M, Lilje L, Davison J, Vasar M, et al. Plant species richness belowground: higher richness and new patterns revealed by next-generation sequencing. Mol Ecol. 2012;21:2004-16.

70. Harrison KA, Bardgett RD. Influence of plant species and soil conditions on plant-soil feedback in mixed grassland communities. J Ecol. 2010;98:384-95.

71. Laliberté E. Below-ground frontiers in trait-based plant ecology. New Phytol. 2017;213:1597-603. 